Jurnal Teknologi, 42(A) Jun. 2005: 27-48

(C) Universiti Teknologi Malaysia

\title{
DESIGN AND DEVELOPMENT OF A PROGRAMMABLE PAINTING ROBOT FOR HOUSES AND BUILDINGS
}

\author{
I. ARIS ${ }^{1 *}$, A. K. M. PARVEZ IQBAL ${ }^{2}$, A. R. RAMLI ${ }^{3} \&$ S. SHAMSUDDIN ${ }^{4}$
}

\begin{abstract}
Nowadays robots are widely used in many applications such as military, medical application, factories, entertainment, automobile industries etc. However, the application of robot is still not widely implemented in construction industry. In construction industry, robots are designed to increase speed and improve the accuracy of construction field operations. It can also be used to do hazardous and dangerous jobs in construction. For example, currently house painting is done manually. This process can be simplified using a special dedicated robot. It is very difficult and troublesome for human being to work in an upright position, especially for painting, cleaning and screwing in the ceiling for a long time. Painting in an upright position is also very dangerous for the eyes. To overcome this difficulty, a programmable painter robotic system is proposed, designed and developed. This paper describes all the processes that are involved in designing and constructing the proposed painter robot. The system is divided into two main parts namely hardware and software. In hardware part, mechanical design, fabrication, electrical and electronics system are described and in software part, control algorithm is explained. The testing results indicate that the performance of the painter robot is better compared with that of using manual painting technique.

Keywords: Painting machine, cartesian robotic system, PLC (Programmable logic controller), electropneumatic system, motor controlling, construction robotics
\end{abstract}

\subsection{INTRODUCTION}

The term robot implies different meaning to different people. Construction robots are ingenious machines that use intelligent control but vary in sophistication. Generally, they are designed to increase speed and improve accuracy of construction field operations. Construction robots are especially helpful where the constructions are done under dirty and dangerous conditions. According to Steward [1] and Stein [2], the Japanese have a liberal interpretation of the term "robot". Their definition includes advanced automation and remote control devices used on the construction site and prefabrication shop. However, there is no consensus on a clear definition of construction robotics [1-5].

The construction industry uses different kind of robot. Generally in the manufacturing field, robots are stationary and the product moves along the assembly

\&2 Department of Electrical and Electronic Engineering,

3 Department of Computer and Communication System Engineering,

4 Department of Mechanical and Manufacturing Engineering,

Faculty of Engineering, Universiti Putra Malaysia, Serdang, 43400 Selangor, Malaysia

*Corresponding author. Email: ishak@eng.upm.edu.my 
line. However, construction robots must move inside and outside the buildings, which are stationary and of a large size. The operating parameters of the robot keep changing and it needs to be reprogrammed with each new condition. That is why digital control with manipulators using coordinate systems to control three-dimensional motion is required for construction robots. Construction robots often handle large loads with components of variable sizes. They must be also able to function under adverse weather conditions including variation in humidity and temperature. Designing construction robots are more challenging compared with those conventional industrial robots. There are now 89 different kinds of single task robots that are used on Japanese construction sites [6]. Single task robots perform a specific job and imitate construction labor. Although single task robots have been successful but these devices are limited because they are not capable of identifying and fixing the problem in real time. Workers are still needed to set up, monitor, and clean the machine. Because of these limitations, the Japanese have been exploring the use of fully automated construction site. According to Moore, contractors using this approach report time savings of 30\% and labor cost reductions of 50\% [7]. The computerised information management system keeps a running inventory of materials, drawings, schedules and volume of completed working real time [8]. Some previous works related to painting machine are described as follows.

The University of Texas has developed a prototype-automated surfaces finishing system for use on large diameter tanks. This system uses a computer-controlled motion module to refinish the vertical exterior walls of a tank. The module is configurable for both blasting and painting and utilises the conventional surface finishing equipment for these processes [9]. This system consists of an aluminum motion module, which rolls on the surface of the tank. The module attaches to the wind girt of a tank via two steel cables. The position and velocity of the module are determined by the amount and speed of the cable splayed through two hoist located at the rear of the module. Squirrel cage assemblies mounted beneath the motion module retain the excess cable from the hoist [10-11]. Another robot for painting the exterior wall was developed by Terauchi [12]. This robot is mounted on equipment, which permits it to move up and down, left and right along the exterior walls of the building. It is computer controlled and activated simply by the operator pressing a switch on the control panel located on the ground. The robot is capable of painting a four square meter wall surface at one time. It is also equipped with sensors, which measure indentations and protrusions in the wall surface, making it possible for it to paint exterior walls with windows, pillars or other indentations or protrusions.

Another painting machine for applying the coatings to elongated thin shaft such as golf club shafts was invented by Bauerle [13]. This specially designed machine can apply precise, uniform coatings to elongated shafts. The conventional painting techniques are not effective in painting the thin elongated shaft. Painting with brushes and rollers requires considerable skill, is time consuming and likely to result in 
uneven coatings with streaks, brush marks, runs, and other irregularities. Spraying also requires considerable skill to produce even coating without runs or uneven areas. In addition, where the shaft is quite narrow, much of the coating material is wasted as over spray passes by the object being painted. In order to solve the coating problem, this machine was developed. Shafts are held by a gripper in a downwardly hanging array. A paint head assembly is moved upwardly over each shaft in sequence to apply a paint coating to a selected portion of the shaft length. The paint head assembly includes a flexible container including a central hole, a vertically moveable edge frame and an expander for changing the area of the hole. Raising the frame forms the container into a bowl like configuration, so that the paint will contact a shaft extending through, and in contact with the hole.

According to Warszawaski and Rosenfeld's [14] economic analysis of performance of multi-purpose interior finishing robot, it can be concluded that the employment of a robot for interior finishing works has considerable potential for productivity improvement on the building site. It appears that economic savings can be also realised from robots employment. Other non-quantifiable benefits which can be also obtained include increased safety, reduction of strenuous and unpleasant tasks, and improved quality of building. Realisation of these benefits depends, however, on very high precision of the building shell and a very high level of materials packaging and handling, and work organization on site.

A painting machine for painting the surgical tubes was invented by Flood [15]. This painting machine includes a chain driven track assembly, which moves the tube pieces held on paint fixtures into a paint chamber for painting and into an oven to dry the paint to the tube.

From the discussion mentioned above, it is clear that the automatic spray painting is feasible for various finishing tasks. In the construction industry, different painting machines, robots and techniques are used for painting the external and internal walls of the buildings and houses. It is also very difficult and troublesome for human to work in an upright position, especially for painting, cleaning and screwing in the ceiling for a long time. Painting in an upright position is also very dangerous for the eyes. To overcome this difficulty, a robotic system which is capable of painting the houses and buildings is proposed and developed. The proposed painter robot has three degrees of freedom (DOF). For $X$ direction, a single-phase induction motor and a chain-sprocket mechanism are used. Two limit switches and two electronic sensors are used to limit the movement in $X$ direction. Another sensor is used to position the robotic arm along the $X$ direction. For $Y$ direction, two limit switches are used to limit the movement in $Y$ direction. Two sensors are used to protect the robotic arm along the $Y$ direction. The single-phase motor with an inverter is utilized to control the speed of the robot in $Y$ direction. For $Z$ direction, a parallelogram structure and a ball-screw mechanism are used in this project. A single-phase brake motor and a photoelectric sensor are used to control the position in $Z$ direction. Two limit switches are used to limit the movement in $Z$ direction. The proposed 
robot is used to paint the ceiling of houses. The paint is automatically sprayed by the robot using pneumatic system.

The software part involves the design and development of the system control software. The system control software is created using FP WIN GR PLC programming software. This software can run on windows. It has its own graphics interface. It is more user friendly compared with a hand-held programmer. This software provides three programming styles: Ladder symbol mode, Boolean, Ladder Mode and Boolean Non-ladder mode [16]. In this system, the ladder symbol mode is used to create the control program of the proposed robot. The Programmable Logic Controller (PLC) is used to control the overall operation of the robot.

\subsection{SYSTEM DESIGN}

The proposed robotic system consists of a personal computer, a controller, input and output devices, actuators and the mechanical structure. A personal computer is needed to develop the program and then download this program into the controller. The PLC is used as the main controller of the robotic system. The input signals from the input devices will be processed in this controller and the resulting output signals will activate the output devices. The actuators are utilised to drive the mechanical robotic structures. Figure 1 shows the processes that are involved in developing this robotic system.

\subsection{MECHANICAL DESIGN}

The mechanical design consists of a positioning module and an end-effector module.

\subsection{Positioning Module}

The design of the positioning module of this painter robot is based on the Cartesian coordinate concept and it is also known as the $X-Y-Z$ coordinate. It is the combination of individual joints where the action must be controlled in order to perform the robotic manipulator in the desired motion cycle. For $X$ and $Y$ modules movement, the chain-sprocket mechanism is used. For $Z$ module movement, the ball-screw mechanism is utilised. Thirty-six aluminium plates are used as links to make the $Z$ axis as parallelogram structures. Two parallelogram structures are connected to each other by the iron rod connector.

\subsubsection{X-axis Module}

The $X$-axis module is the first step of this project to be fabricated according to the proposed design. Figure 2 shows the $X$-axis module of the system. The description of the $X$-axis module includes the following: 
The $X$-axis module is fabricated to provide the motion of the robotic system in the $X$ direction. The base frame, which is included in the $X$-axis module, is fabricated using a hollow iron bar. The hollow bar is selected for its low weight and adequate strength. Four wheels are attached at the four bottom corners of the frame. Two of the four wheels are caster wheels and the remainings are unidirectional wheels. The objective of providing the wheels is to give mobility to the whole system. Two slider guide ways and four runner blocks are mounted along the $X$-axis at the top of the frame. These are the main parts of the $X$-axis module. Specially designed mountings are attached to the two opposite side runner blocks. These mountings are designed to hold the $Y$-axis module. The mild-steel made mountings are attached to the driving chain and are designed to hold the shafts, bearings and the motor of the $Y$-axis module and to attach the driving chain of the $X$-axis module. Four pillow bearings are attached with the frame to hold the two shafts of the $X$-axis module. Each shaft consists of two sprockets at its opposite end. The sprockets are provided to drive the

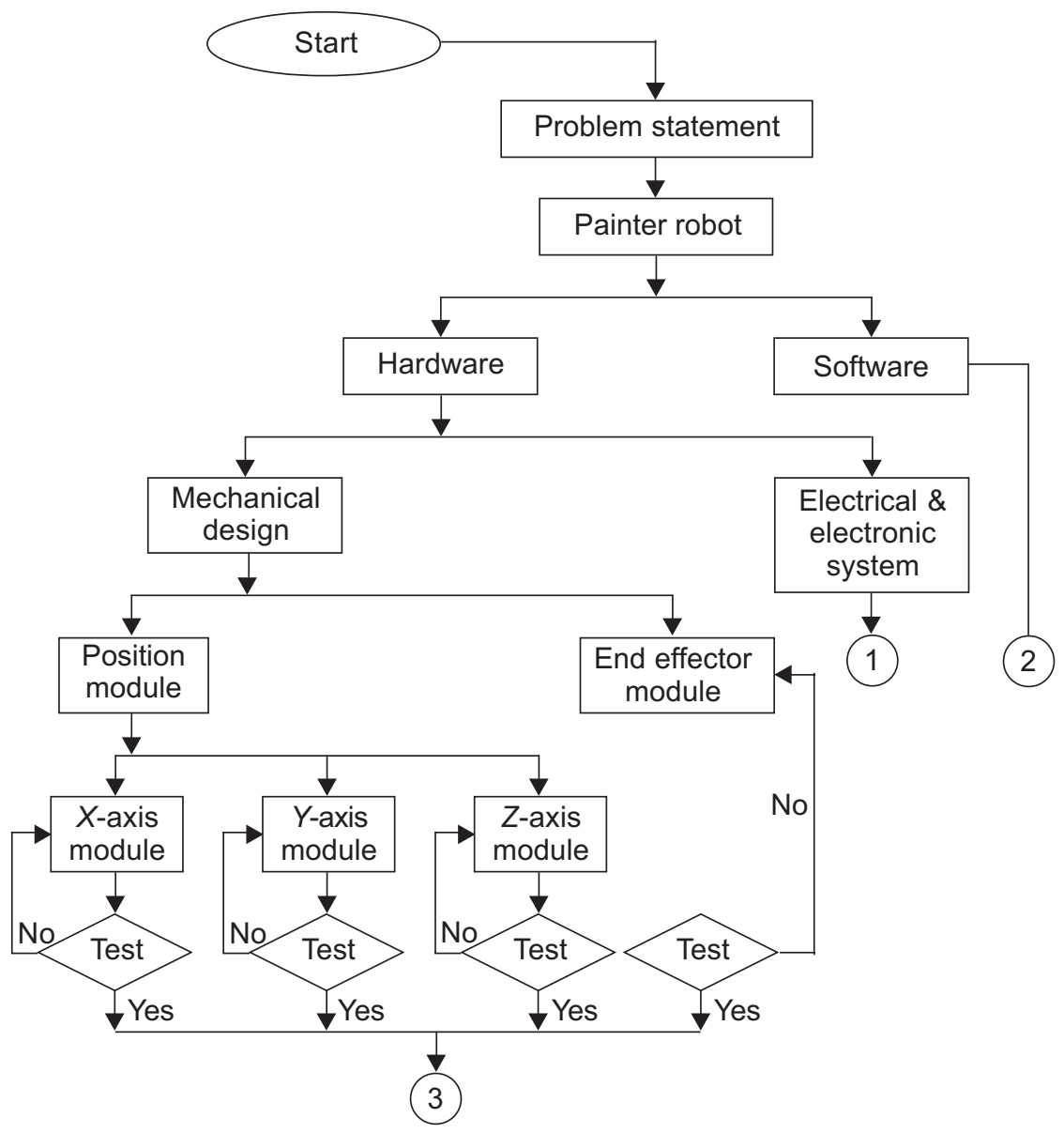

Figure 1 The process that are involved in developing the robotic system 


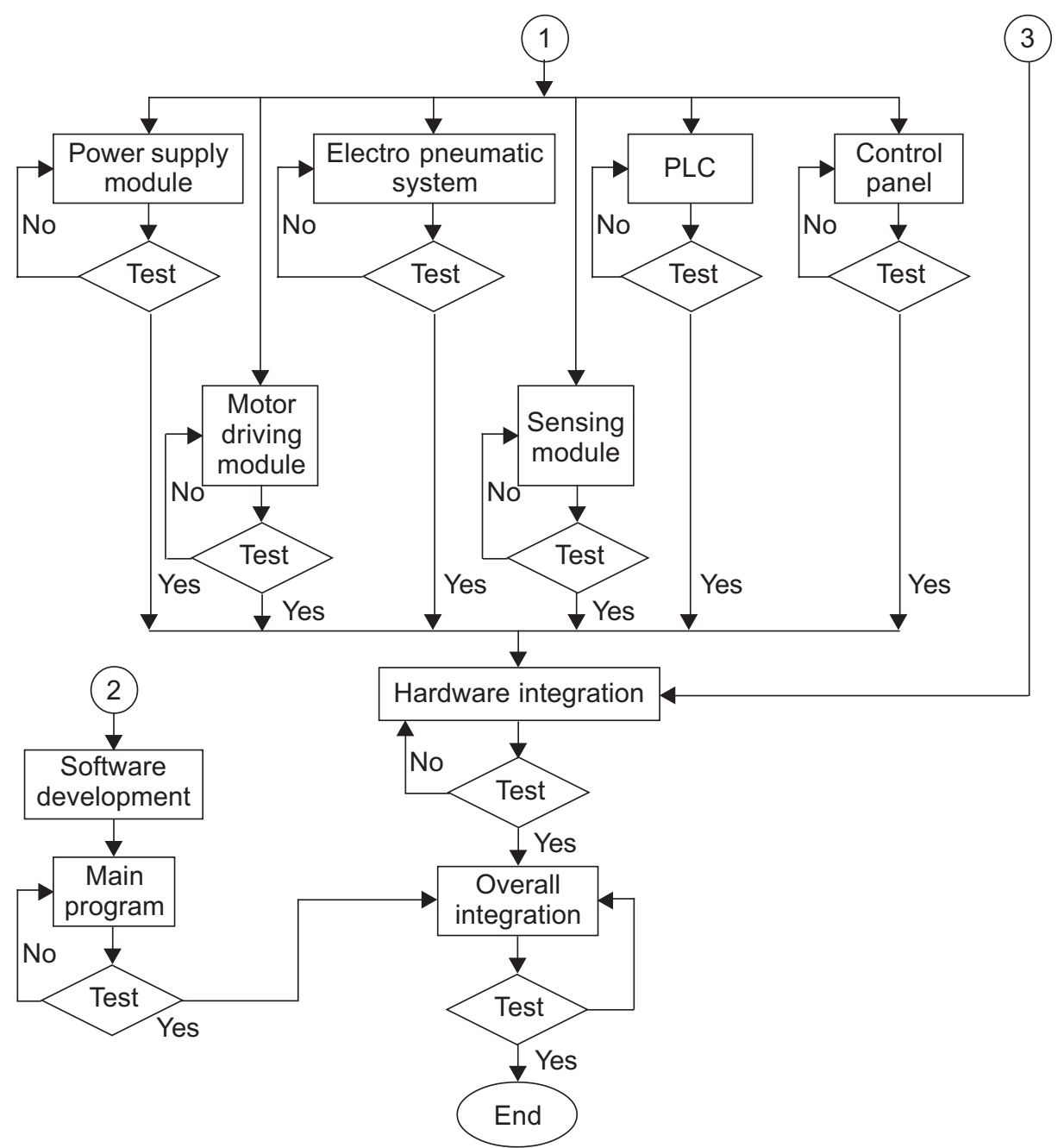

Figure 1 The process that are involved in developing the robotic system (cont.)

chains. Two chains are used to drive the $X$-axis module. Four tensioners are attached in the frame to adjust the chains of the $X$-axis module. The sprocket bearing attachment is mounted at the end of each tensioner. This sprocket can freely rotate and mesh with the chain. The tensioners are mounted in the frame so that they can adjust their angle by loosening the screws. After adjusting the angle, they can be fixed by screwing. The angle adjustment is necessary for adjusting the chain. A single-phase AC induction motor is mounted with a frame to drive the $X$-axis module. The motor is coupled with the shaft by the flexible coupling. To select a proper motor, it is very important to know the amount of torque, which can drive the $X$-axis module. The motor, which is used for the $X$-axis module, will have to carry the total systems. Two limit switches, three inductive proximity sensors and one photoelectric sensor are used to 


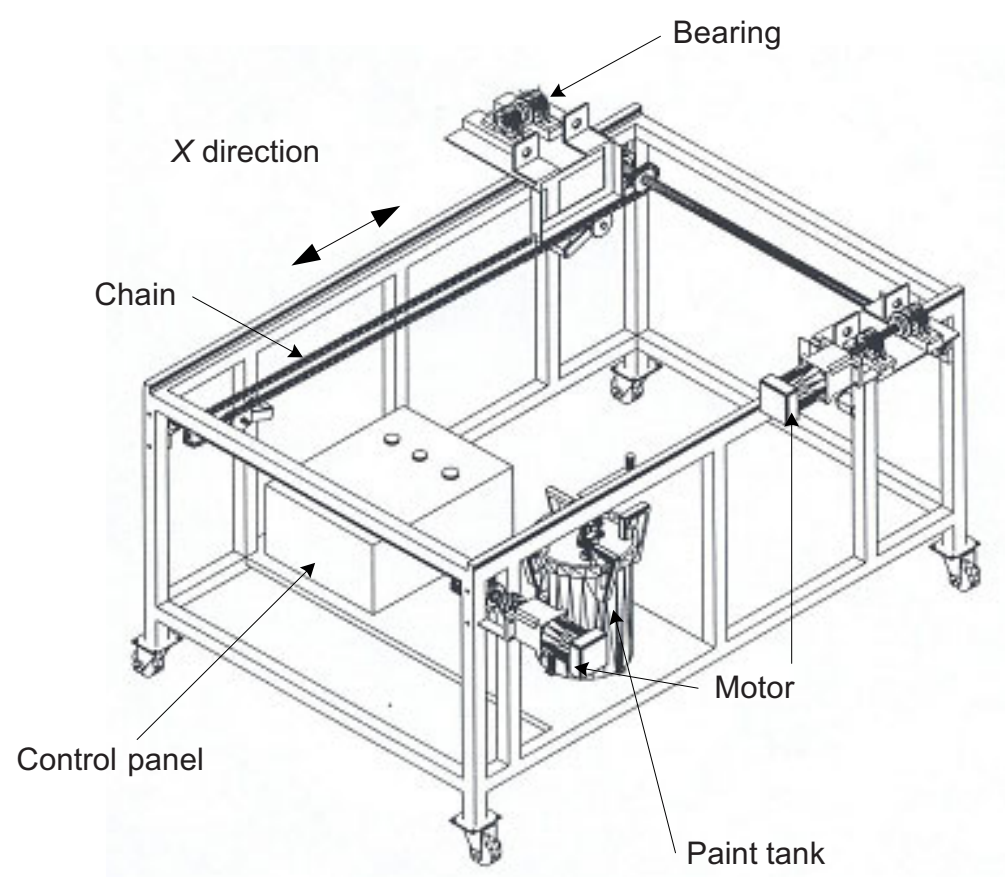

Figure $2 X$-axis module of the painter robot

control the $X$-axis movement. In order to determine the suitable motor size, it is important to calculate the torque of the $X$-axis module. The process of calculating the torque is described as follows:

$$
\begin{gathered}
T_{\text {Total }}=T_{\text {load }}+T_{\text {sprocket }}+T_{\text {Chain }}+T_{\text {Motor }}+T_{\text {Friction }} \\
=\frac{1}{g}\left(J_{\text {Load }}+J_{\text {sprocket }}+J_{\text {Chain }}+J_{\text {Motor }}\right) \frac{\omega}{t}+T_{\text {Friction }} \\
J_{\text {Load }}=W_{L} R^{2} \\
J_{\text {sprocket }}=\frac{W_{S} R^{2}}{2} \\
J_{\text {Chain }}=W_{c} R^{2} \\
T_{\text {Friction }}=F R \\
\omega=\frac{V}{R}
\end{gathered}
$$


The above formulae are taken from Parker motion and control catalog [17]. Using the above equations and considering all design factors, the total torque for the $X$ - axis module is found as:

$$
\begin{aligned}
T_{\text {Total }} & =\frac{1}{9.81}\left(0.298+2.66 \times 10^{-4}+0.0254+0.0647\right)+0.1608 \\
& =0.608 \mathrm{~N}-\mathrm{m}
\end{aligned}
$$

After getting the value of the torque, the motor for $X$-axis module was determined. A $0.95 \mathrm{Amp}, 240 \mathrm{~V}$ single phase AC induction motor was selected for this application. The general velocity profile and torque-speed curve are shown in Figures 3 and 4. The two limit switches are used to provide the maximum limit and the inductive proximity sensors are used to control the positioning of the $X$-axis module. The photoelectric sensor is used to protect the total system from obstacles in the work place when painting process is in progress.

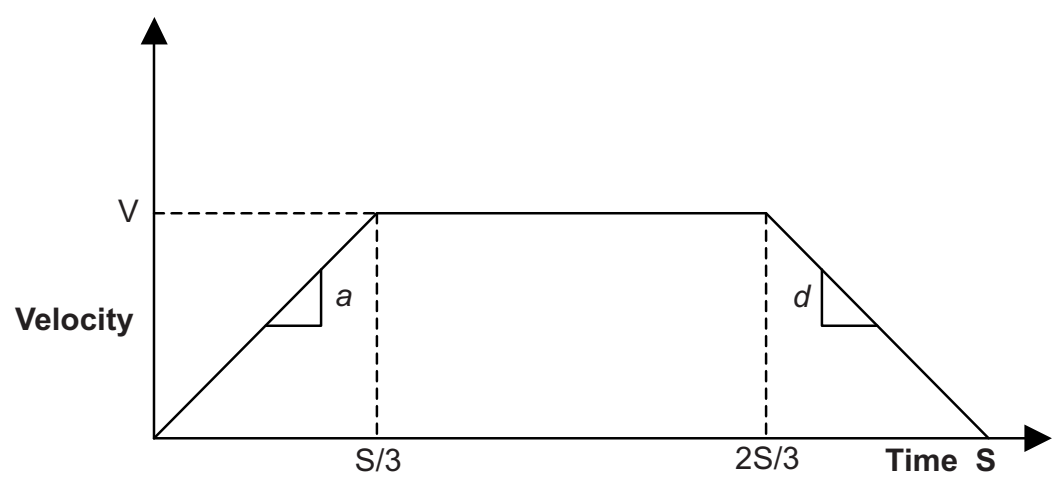

Figure 3 General velocity profile of motor

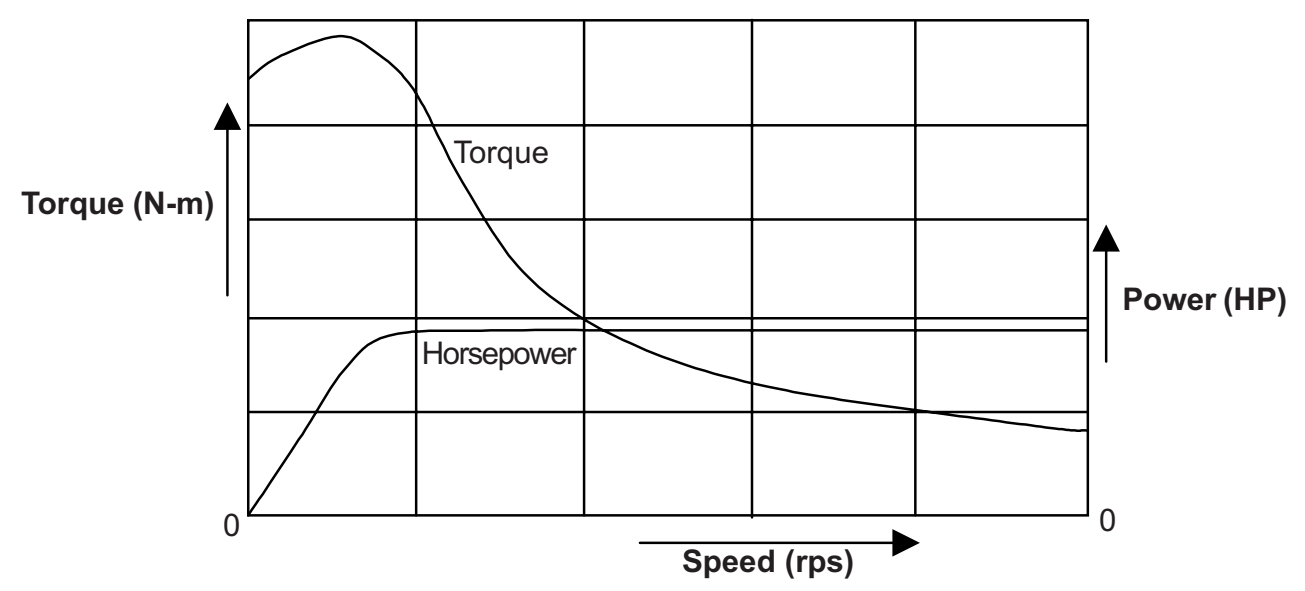

Figure 4 Torque-speed curve of the motor 


\subsubsection{Y-axis Module}

The $Y$-axis module is depicted in Figure 5. The chain sprocket mechanism and motor are used to carry the $Z$-axis module and the end-effector module in the $Y$ direction. To make the $Y$ direction movement smooth and frictionless, hardened guide rods and linear bushings are provided. The design of the $Y$-axis module can be divided into two parts, namely the hardened guide rod selection and the motor torque calculation. By analysing the bending stress, it is possible to select a proper hardened guide rod. The process for selecting the hardened guide rod is as follows:

$$
\begin{gathered}
\delta_{d}=\frac{M Y}{J} \\
M=W_{L} \times \frac{l}{2}=m_{L} \times g \times \frac{l}{2} \\
\mathrm{Y}=\frac{d}{2} \\
J=\frac{\pi}{4}\left(\frac{d}{2}\right)^{2} \\
\delta_{d}=\frac{\delta_{u} o r \delta_{y}}{F S}
\end{gathered}
$$

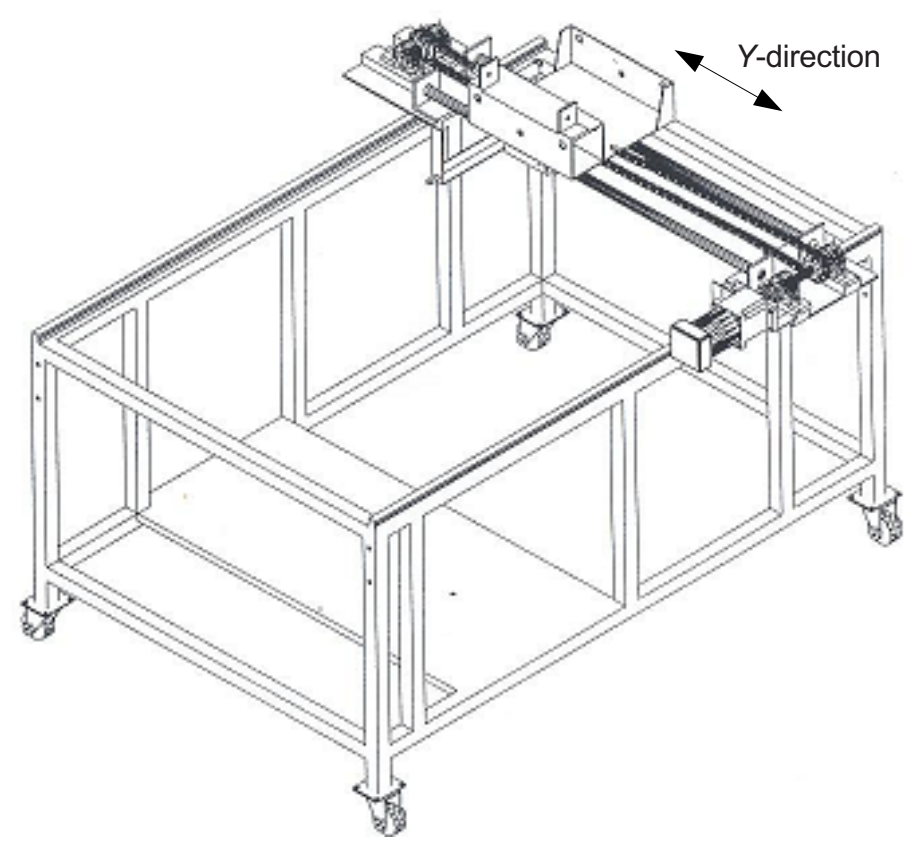

Figure $5 \quad Y$-axis module the painter robot 
After using the above formulae and considering all design factors, the diameter of the guide rod was calculated to be of $24.5 \mathrm{~mm}$. The system uses two hardened guide rods as a guide for smooth running of the $Y$-axis module, the $Z$-axis module and the end-effector module. Therefore, the diameter of each guide rod is at least half of the calculated value. Figure 6 shows the free body diagram and cross sectional area of the guide rod.

The $Y$-axis module is fabricated to provide the motion in the $Y$ direction. Two stainless steel guide rods and two linear bushings are provided in the $Y$-axis module. These are the main parts required to provide the motion in the $Y$ direction. The stainless steel guide rods are attached to the mountings, which are mounted to the two opposite side runner blocks in the $X$-axis module. To avoid the sliding of the guide rod during operation, slots and circular locks are provided at both ends of the guide rods. When the guide rods are adjusted to the mountings of the $X$-axis, these slots and circular locks fix the guide rods to the mountings. Two flange bushings are used in the $Y$-axis module. The flanges are used with bushings to attach the bushing with other body. These two flange bushings are inserted to the two fixed stainless steel guide rods and provide the $Y$-axis motion. A specially designed mounting is attached to the flange bushing. This mounting is designed to hold the $Z$-axis module. It can also be attached to the roller chain of the $Y$-axis module. Four pillow bearings and two shafts are used to carry the mountings along the $Y$-axis direction. The roller chain and sprocket are used for this movement. An AC induction motor, which has similar rating as that one used for the $X$ direction, is mounted with a frame to drive the $Y$-axis module. The motor is coupled with the shaft by a flexible coupling. The motor for the $Y$-axis module is selected using the process, which is mentioned in the $X$-axis module. Two limit switches are used to control the maximum limit of movement of the $Y$-axis module in both forward and reverse directions. Two photoelectric sensors are used to protect the system during the operation, especially when the robot runs in both forward and reverse movements in the $Y$ direction.

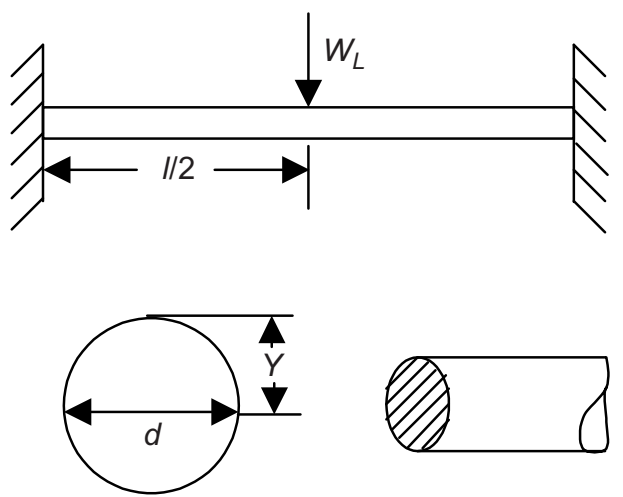

Figure 6 Free body diagram and cross sectional area of the guide rod 


\subsubsection{Z-axis Module}

Figure 7 shows the $Z$-axis module that is used in the painter robot. The $Z$-axis module is fabricated to provide the motion in $Z$ direction for the robot. Thirty-six aluminium plates are used to make this module. The dimensions of each plate are the same. The plates are linked to each other in such a way that they can form a zigzag ladder. There are two zigzag ladder structures in the $Z$-axis module, which are parallel to each other. These structures are linked to each other by mild-steel pins attached at the joints. The gap between each parallel structure is $20.5 \mathrm{~cm}$. These parallelogram structures are made in this way to avoid inclination. The bottom end of the parallelogram structures is hinged to a mounting, which is attached to the $Y$ axis module. One part of the bottom end is hinged to the mounting but this part is not movable. The other part of the bottom end is hinged and this part can move back and forth. When this movable part moves forward, the structures go up and when the movable parts moves backward, the structures go down. Four deep-grooved ball bearings are used to make the rotation of the bottom part of the parallelogram structure smooth. These four bearings are fixed to the ends of four bottom plates which ends are used for making hinges. Two linear bushings and two stainless steel guide rods are used to provide linear motion of the movable bottom part of the parallelogram structures. The ball-screw mechanism is used to drive the movable bottom part of the structure. The special arrangement is provided to hold a ballscrew nut to the movable bottom part of the structure. The ball-screw is mounted to the two flange bearings, which are attached to the mounting of the $Y$-axis module. When the ball-screw rotates clockwise or counter clockwise, the nut and the movable bottom part move forward or backward. An induction reversible brake motor is used to drive the ball-screw. This motor is coupled with the ball screw by a flexible

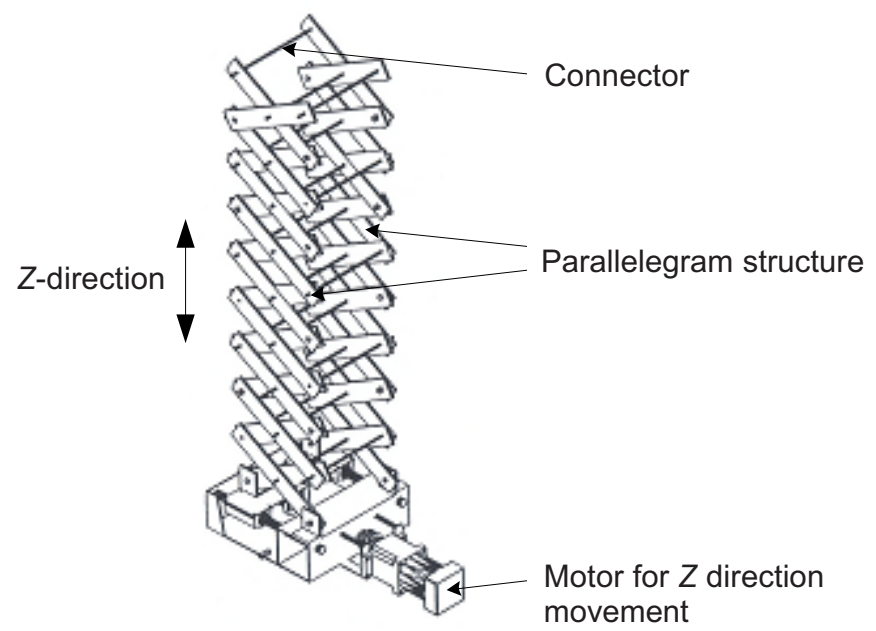

Figure $7 \quad Z$-axis module of the painter robot 
coupling. To select a motor for the $Z$-axis module, it is important to know the amount of torque required to drive the ball-screw. The process to calculate the torque is as follows:

$$
\begin{gathered}
T_{\text {Total }}=T_{\text {Friction }}+T_{\text {Acceleration }} \\
T_{\text {Friction }}=\frac{F}{2 p e} \\
\omega=2 p V \\
T_{\text {Acceleration }}=\frac{1}{g}\left(J_{\text {Load }}+J_{\text {ball-screw }}+J_{\text {motor }}\right) \frac{\omega}{t}
\end{gathered}
$$

By using the above equations and considering the design factors, the torque of the motor of the $Z$-axis module was determined as $0.069 \mathrm{~N}-\mathrm{m}$. After getting the value of the torque, it is clear that the torque of the $Z$-axis motor must be higher than the calculated value to ensure a smooth movement. One photoelectric sensor and twolimit switches are used to control the movement of the $Z$-axis module. The photoelectric sensor is used to detect the ceiling position and two limit switches are used to control the maximum movement along the $Z$-axis.

\subsection{End-Effector Module}

The end-effector module is designed in such a way that it can hold a spray gun and operate the spray gun during the operation. A single acting pneumatic cylinder is attached to the lever. The lever can move up and down by the actuation of a cylinder. Figure 8 shows the proposed design of the end-effector module. Two hosepipes are attached to the spray gun. One of them is directly connected to a paint tank and the other is connected to an air compressor. The paint tank is a pressure vessel and is directly connected to the air compressor. The paint is moved through the hosepipe by air pressure. The air pressure that is used by the robotic system to perform the painting activity is $10 \mathrm{bar}$. This air pressure is kept constant throughout the operation to achieve the standard quality paint.

\subsection{ELECTRICAL AND ELECTRONIC SYSTEM}

There are six main parts in the electrical and electronic system of a painter robot. They are a power supply module, a main controller PLC, a sensor module, an electro-pneumatic system, an AC induction motor drive system and a control panel. A proper distribution of power supply is required to activate the components of the system. The AC and DC voltages are supplied and distributed to the robotic system as depicted in Figure 9. A supply of $24 \mathrm{~V}$ DC voltage is required for most of 


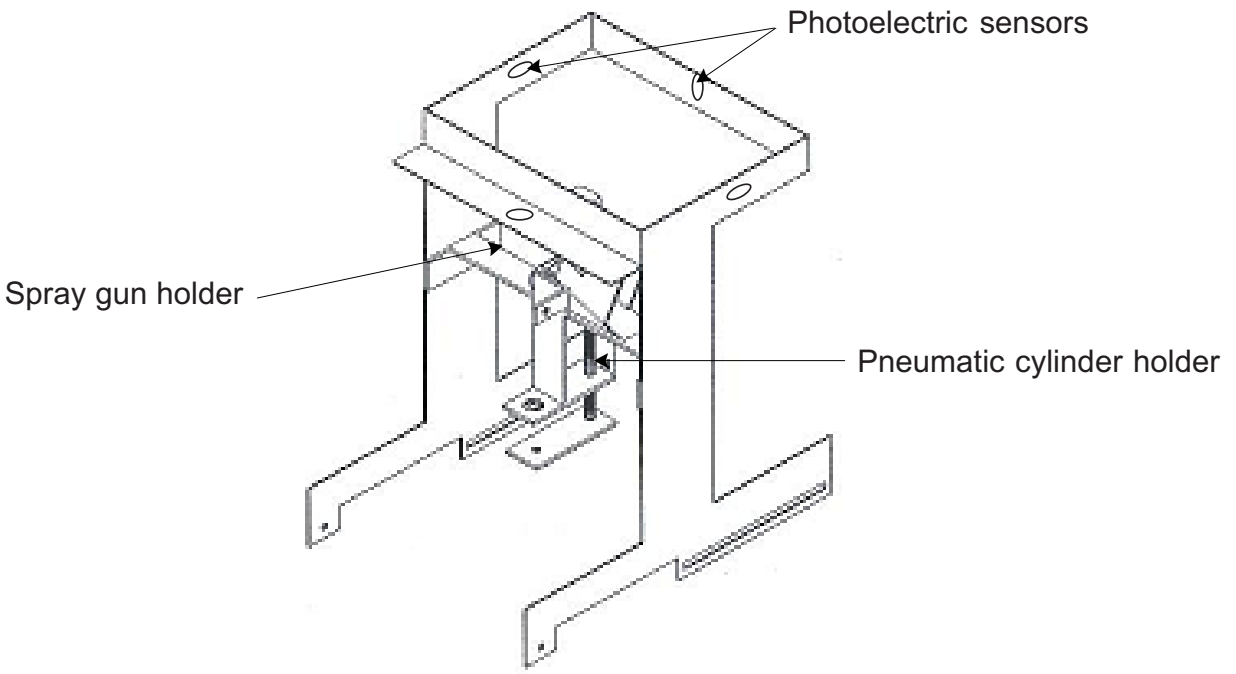

Figure 8 End-effector module (paint spray gun holder) of the painter robot

the electrical and electronic components such as PLC, sensors, pneumatic valve, limit switches etc. In this system, a commercial switching power supply unit is used to convert the $\mathrm{AC}$ voltage from the main source to $24 \mathrm{DC}$ voltage. The input of $240 \mathrm{AC}$ voltage is reduced to $36 \mathrm{~V} \mathrm{AC}$ and then rectified and regulated to $24 \mathrm{~V}$ DC. The output $24 \mathrm{~V} \mathrm{DC}$ is distributed to activate the main controller, optical sensors, pressure sensors, inductive proximity sensors, limit switches, start/stop button, indication light and motor driven relay. The AC induction motors are connected directly to the main supply. Figure 10 depicts the inside of the control panel of a painter robot.

\subsection{System Protection}

Four photoelectric sensors, three inductive proximity sensors and six limit switches are used to protect a painter robotic system from obstacles in the work environment. For the $X$ direction, inductive proximity sensors are used to change the position of the robotic system along the $X$-axis and provide the maximum running length within the $X$-axis. To ensure the protection of the system in the $X$-direction, one photoelectric sensor and two limit switches are provided in the $X$-axis module. When the system changes its position along the $X$-direction, during this time, if the sensor detects any obstacle, it will stop the total system. The total system can be stopped again if the system touches the limit switches. The system can be initialised to return to its home position by using the reset button. For the $Y$-direction, two photoelectric sensors and two limit switches are used to protect the system and limit the movement of the 


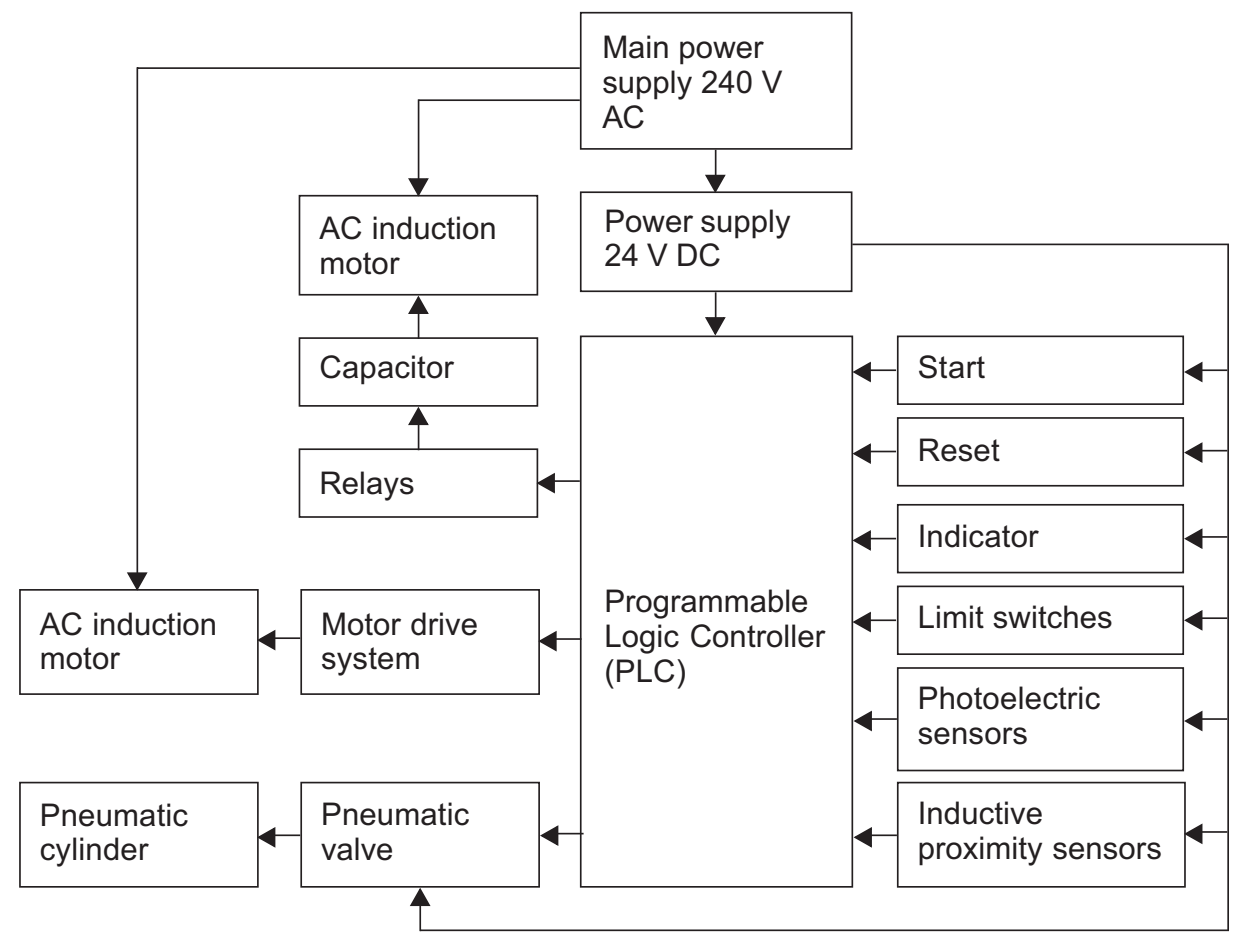

Figure 9 Power distribution system of the painter robot

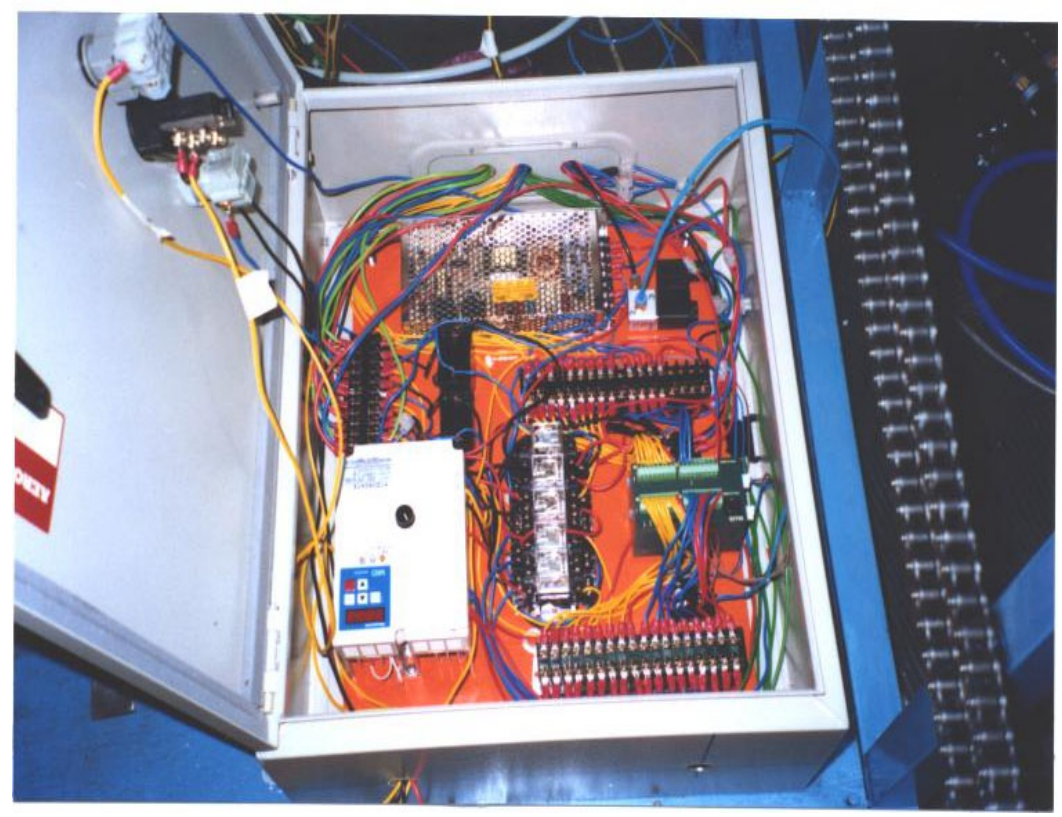

Figure 10 The inside of a control panel of a painter robot 
system along the $Y$-direction. Painting operation will be performed in this direction. During this operation, if the sensors detect any obstacle in forward or reverse direction, the painting process will be stopped and the system will be changing its position along the $X$-direction. After changing the position, the painting process will start again automatically. For the $Z$-direction, one photoelectric sensor and two limit switches are used to detect the ceiling and limit the movement in the $Z$-direction. The maximum range of movement of the robotic system along the $Z$-direction is $1.83 \mathrm{~m}$. If the system crosses the maximum limit, the limit switch will stop the total system. In the reverse direction, along the $Z$-axis, when the system reaches its initial position, another limit switch is used to stop the movement of the system. Within this range of movement, the proposed robotic system is capable in performing the painting operation. An AC induction brake motor is used to keep the robotic system in an up-right condition along the $Z$-direction. Figure 11 illustrates the sensor detection system.

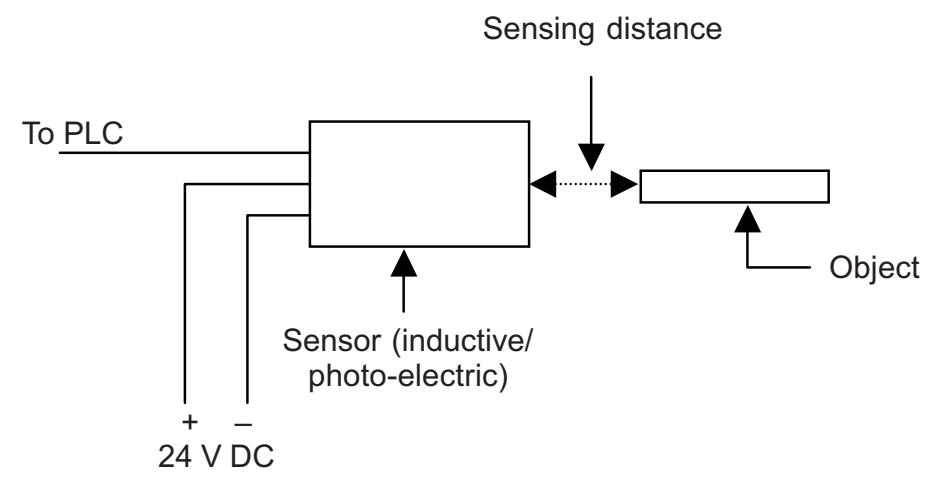

Figure 11 The detection system of sensor

\subsection{SOFTWARE DEVELOPMENT}

The PLC is used to control the operation of the proposed robot. A new program to control the PLC was written. After the program is loaded, it will go into a run mode. At that time, it can check the input COM ports and solve the user program ladder logic instructions. Figure 12 shows the programming environment and ladder diagram of FP WIN GR software. According to the input instruction, it can control the output COM ports and their associated devices. Table 1 shows the input and output mapping of the system. A personal computer and PLC programming software are used to develop the program. The program is downloaded to the PLC via the communication cable through the RS232 port. The software which is used in this project is called the FPWIN GR. Before starting the project, it is important to do the planning for the user program. The planning is needed to create a workable, reliable, and efficient 


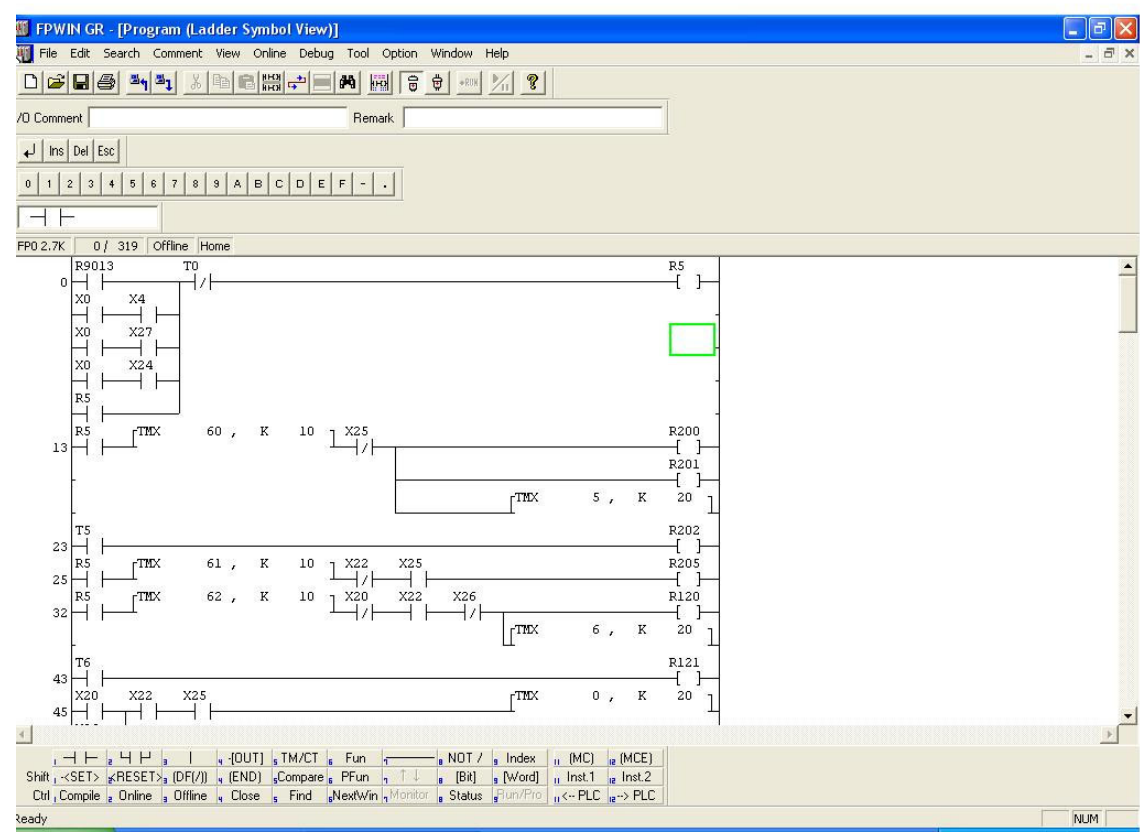

Figure 12 Programming environment and ladder diagram of FP WIN GR software

Table 1 Input and output mapping of the Programmable Logic Controller of the proposed painter robot

\begin{tabular}{llll}
\hline Input port & Device & Output port & Device \\
\hline $\mathrm{X}_{0}$ & Start button & $\mathrm{Y}_{0}$ & Motor brake \\
$\mathrm{X}_{1}$ & Reset button & $\mathrm{Y}_{1}$ & Motor for $Z$ axis \\
$\mathrm{X}_{2}$ & Upper sensor & $\mathrm{Y}_{2}$ & Motor for $Z$ axis \\
$\mathrm{X}_{3}$ & Forward sensor & $\mathrm{Y}_{3}$ & Pneumatic Valve \\
$\mathrm{X}_{4}$ & Side sensor & $\mathrm{Y}_{4}$ & Motor for $Y$ axis \\
$\mathrm{X}_{5}$ & Backward sensor & $\mathrm{Y}_{5}$ & Motor For $Y$ axis \\
$\mathrm{X}_{6}$ & X axis positioning sensor 1 & $\mathrm{Y}_{20}$ & Motor for $X$ axis \\
$\mathrm{X}_{7}$ & Pressure sensor & $\mathrm{Y}_{21}$ & Motor for $X$ axis \\
$\mathrm{X}_{20}$ & Sensor for home position & $\mathrm{Y}_{22}$ & Indicating light \\
$\mathrm{X}_{21}$ & X axis positioning sensor 2 & & \\
$\mathrm{X}_{22}$ & Limit switch 1 & & \\
$\mathrm{X}_{23}$ & Limit switch 2 & & \\
$\mathrm{X}_{24}$ & Limit switch 3 & & \\
$\mathrm{X}_{25}$ & Limit switch 4 & & \\
$\mathrm{X}_{26}$ & Limit switch 5 & & \\
$\mathrm{X}_{27}$ & Limit switch 6 & & \\
\hline
\end{tabular}


PLC program. The following sequences are used to develop the program as illustrated in Figure 13.

- Turn on the air compressor.

- Turn the start button on.

- From the home position, the $Z$-axis motor turns on.

- The robotic arm moves up until the upper sensor detects the ceiling.

- The upper sensor detects the ceiling and stops the $Z$-axis motor.

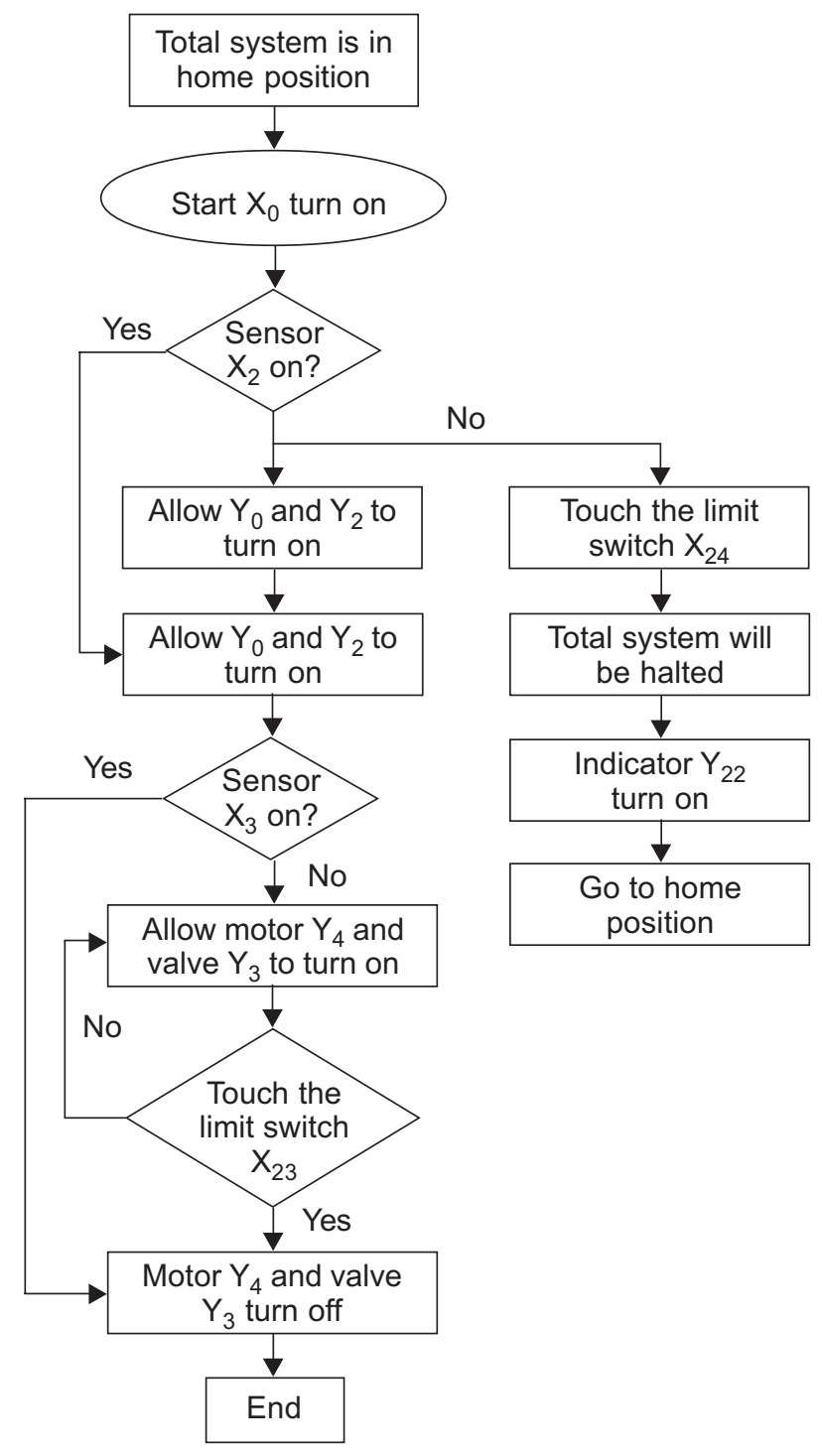

Figure 13 Partial flowchart of sequence of operations 
- $\quad$ The pneumatic valve and the $Y$-axis motor turn on and painting will start until the forward sensor detects or the limit switch touches the robotic arm.

- The forward sensor detects and turns off the pneumatic valve and the $Y$-axis motor.

- The limit switch touches the robotic arm and stops the pneumatic valve and the $Y$-axis motor.

- The $X$-axis motor turns on and moves the system along the axis until the side sensor and the sensor along the $X$-axis detect it.

- $\quad$ The sensor along the $X$-axis detects the position and stops the $X$-axis motor.

- $\quad$ The pneumatic valve and the $Y$-axis motor turn on and painting will start again until the back sensor detects or the robotic arm touches the limit switch.

- The back sensor detects and stops the pneumatic valve and the $Y$-axis motor.

- The limit switch touches and stops the pneumatic valve and the $Y$-axis motor.

- $\quad$ The $X$-axis motor turns on and moves the system along the $X$-axis from the previous position to forward position until the sensor along the $X$-axis and the side sensor detects it.

- $\quad$ The sensor along the $X$-axis detects the position and stops the $X$-axis motor.

- The side sensor detects the beam or other unwanted element, and stops the total system. In that case, it is necessary to initialise the system.

The above sequences are repeated to complete one position. After completing the painting process, the robotic arm returns to its home position by maintaining the following sequences:

- $\quad$ After touching the limit switch, the $Y$-axis motor and the pneumatic valve will be stopped.

- $\quad$ The $Z$-axis motor turns on and moves the $Z$-axis to its initial position.

- The $Y$-axis motor turns on and moves the robotic arm in a backward position.

- The $X$-axis motor turns on in the reverse direction and moves the total system to its home position.

\subsection{SYSTEM INTEGRATION}

The painter robot is created for painting houses and buildings. The fully automatic painter robot has a 3 DOF Cartesian movements and a PLC is used to control the painter robot. The summary of the specifications of the completed painter robotic 
Table 2 Specification of the proposed painter robot

\begin{tabular}{ll}
\hline Type of robot & Cartesian \\
\hline Robotic control & Automatic \\
Degrees of freedom & 3 \\
Working envelope & $(84 \times 72 \times 122) \mathrm{cm}^{3}$ \\
Linear $X$-axis speed & $30 \mathrm{~mm} / \mathrm{sec}$ \\
Linear $Y$-axis speed & $35 \mathrm{~mm} / \mathrm{sec}$ \\
Linear $Z$-speed & $30 \mathrm{~mm} / \mathrm{sec}$ \\
End effector actuation & Pneumatic \\
Type of controller & Programmable logic controller \\
Software & PF WIN GR \\
\hline
\end{tabular}

system is illustrated in Table 2. To make the robot more intelligent, four photoelectric sensors and two inductive proximity sensors are used in this system. The degrees of freedom involve the $X$-axis, the $Y$-axis, the $Z$-axis and the end effector lever movements. The system has the ability to work in a rectangular envelope. The area of the working envelope depends on the length of the slider guide, ball-screw and the positions of the limit switches and the limit sensors. These limit switches and sensors limit the movement of the motors for safety purpose. The linear motion of the $X$-axis and the $Y$-axis depends on the trigger signals that drive the induction motors, the chain and the sprocket drive. The motion of the $Z$-axis depends on the trigger signal and also the pitch of the ball-screw. The $X$-axis and $Z$-axis modules are able to handle a speed of $30 \mathrm{~mm} / \mathrm{sec}$, while the $Y$-axis module is capable of handling the speed ranging from $1 \mathrm{~mm} / \mathrm{sec}$ to $35 \mathrm{~mm} / \mathrm{sec}$. The speed of the $Y$-axis module can be adjusted depending on the quality of paint. Figures 14 and 15 show a 3-D view of the robotic system and a prototype of the painter robot respectively.

\subsection{PAINTING PERFORMANCE TEST}

After integrating the total system, the painting test was performed. By observation, it can be seen that the painting quality depends on the air pressure, the paint and air ratio, and the speed of the $Y$-axis motor. The paint and air ratio can be adjusted by the adjusting screw of the spray gun. The air pressure, which was used to perform the painting was 10 bars. The speed of the $Y$-axis motor can be adjusted by an inverter. During the painting operation, $46 \mathrm{~m}^{2}$ wall surface was painted by the painter robot. To paint this area, this painter robot took 3.5 hours. The painting quality was smooth and consistent. To manually paint the same area of the wall surface, it takes 1.5 more time than the robotic system and the painting quality is not so smooth. The total costs for painting manually and using robot are US\$139.5 and US\$94.25, 


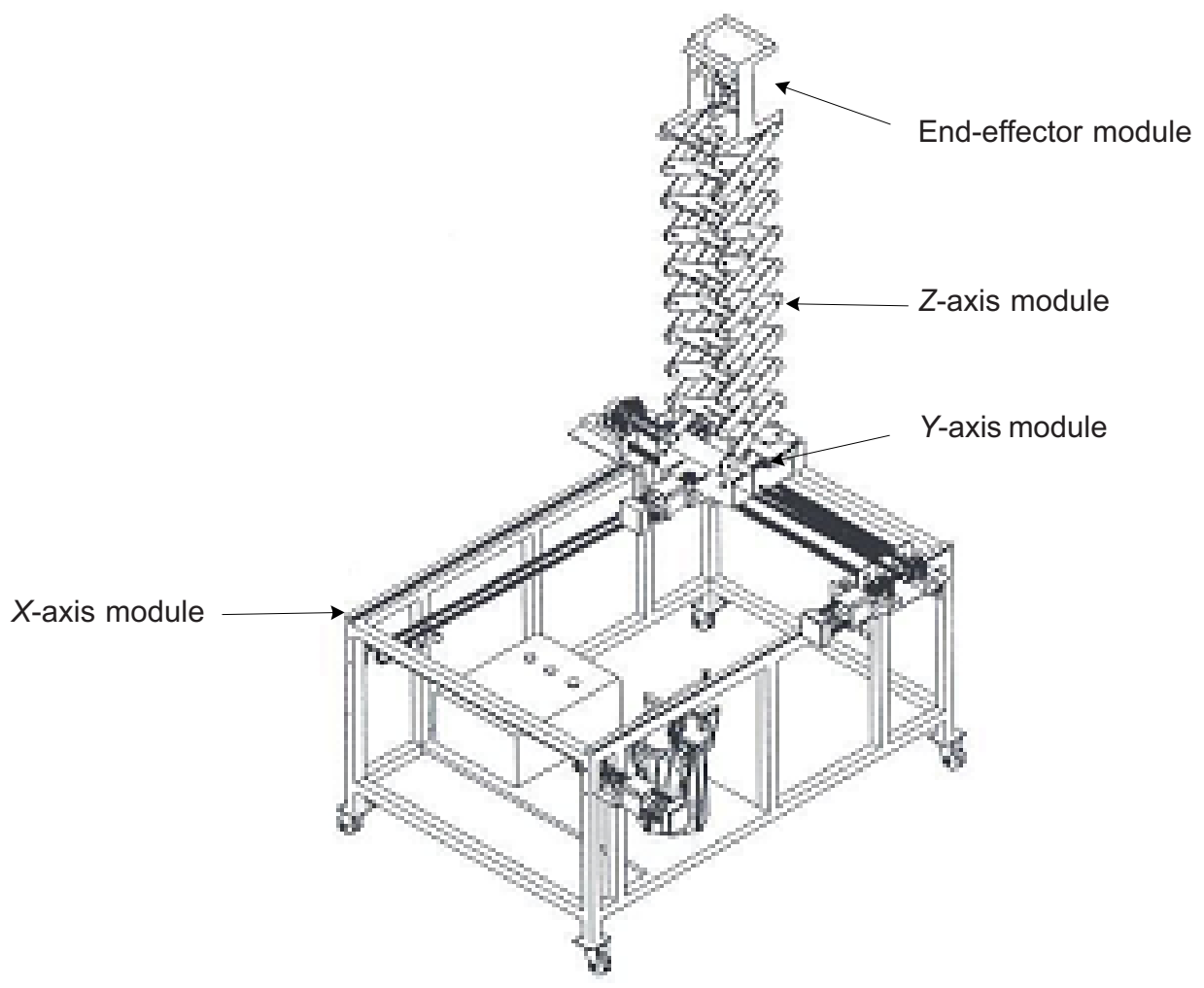

Figure 14 A 3-D view of the painter robot

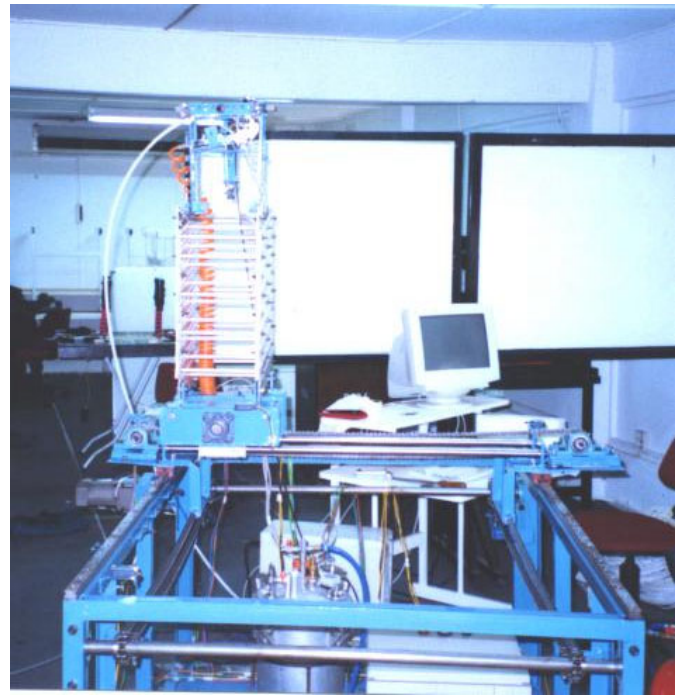

Figure 15 The painter robot demonstrates its capability 
respectively for $46 \mathrm{~m}^{2}$ area. From this comparison, it can be seen that the use of painting robot is more convenient than manual painting.

\subsection{CONCLUSION}

The painter robotic system has achieved optimum benefits with regard to reliability, safety appearance, and ease of use. All the objectives set up for this system have been achieved successfully.

In terms of mechanical design, the $X$-axis, the $Y$-axis, the $Z$-axis module and the end-effector module were designed and fabricated properly. All motor mountings and couplings were properly adjusted. All the prismatic joints were developed successfully.

In terms of electrical and electronic systems, the power distribution module, the sensor module, the electro-pneumatic system, the AC induction motor driving system and the control panel were developed successfully.

In terms of software development, the author had written a control program for the painter robot. This was indicated by the performance of the painter robot. Each movement of the painter robot was successfully controlled by the control program. It can be reprogrammed easily to cope with any changes in the process.

A conclusion can be made that the painter robotic system had been successfully created to solve the problem of working in an upright position, which is very troublesome, boring, unhealthy and harmful to a human being if the working period is long.

\section{ACKNOWLEDGEMENTS}

The authors would like to acknowledge the Institute of Advanced Technology (ITMA) and the Faculty of Engineering of Universiti Putra Malaysia for providing some financial assistance. The authors would also like to thank the staffs of Engineering Faculty of University Putra Malaysia for providing the equipments to carry out the project.

\section{REFERENCES}

[1] Seward, D. 1992. Robots in Construction. Conference Report. Industrial Robot. 19(3): 25-29.

[2] Stein, J. V. M. Gotts, and B. Lahidji. 2002. Construction Robotics. Eastern Michigan University.

[3] Meystel, A. M., and J. S. Albus. 2002. Intelligent System: Architecture, Design and Control. John Wiley and Sons, Inc.

[4] Keramas, J. G. 1999. Robot Technology Fundamentals. Delmar Publisher.

[5] Kao, B. C., and F. Golnaraghi. 2003. Automatic Control System. John Wiley and Sons Inc.

[6] Cousineau, L., and M. Nobuyasu, 1998. Construction Robotics: The Search for New Building Technology in Japan. Reston, VA: ASCE press.

[7] Moore, W. 1999. Working Smarter with Automation. Construction Equipment. 99(4): 44-50.

[8] Yamazaki, Y. M. 1998. The Smart System: An Integrated Application of Automation and Information Technology in Production Process. Computers in Industry. 35(1): 87-99. 
[9] Dailey, C. M., A. E. Traver, G. L. Wesley, C. T. Haas, and J. T. O'Connor. 1993. Field Testing of an Automated Surface Finishing System for Large Diameter Storage Tanks. Automation and Robotics in Construction X. 431-438.

[10] Wesley, G. L. 1991. The Design and Construction of an Automated Surface Finishing Machine for Large Petrochemical Storage Tanks. Thesis. University of Texas.

[11] Freund, M. A. 1991. The Navigation and Control of an Automated Surface Finishing Machine for Steel Petrochemical Storage Tanks. Thesis. University of Texas.

[12] Terauchi, S., T. Miyajima, T. Miyamoto, K. Arai, and S. Takizawa, 1993. Development of an Exterior Wall Painting Robot Capable of Painting Walls with Indentations and Protrusions. Automation and Robotics in Construction X. 285-293.

[13] Bauerle, B. A. 1998. Apparatus for Painting Elongated Thin Shafts. United States Patent. Patent No. 5820676.

[14] Warszawski, A., and Y. Rosenfeld. 1993. Feasibility Analysis of Robotized vs. Manual Performance of Interior Finishing Tasks. Automation and Robotics in Construction X. 383-388.

[15] Flood, J. R. 1993. Surgical Tube Painting Machine. United States Patent. Patent. No. 5209181.

[16] PLC (FP0) Training Manual 2002. Intellogic Technology Privet Limited. Malaysia.

[17] Step Motor and Servo Motor Systems and Controls. Catalog, Parker Motion and Control. 1996/1997. 